Of the 71 patients, $26(37 \%)$ exhibited a peak VO2 <84\% predicted. 11 patients $(15.5 \%)$ developed breathlessness on exertion, 7 (9.9\%) exhibited a fall in blood pressure below baseline and $3(4.2 \%)$ developed ST depression. 18 patients $(25.3 \%)$ had a peak VO2 $<84 \%$ predicted, but without symptoms, fall in blood pressure or ST depression. There was a significant correlation between peak VO2 and AVA $(r=0.24$, $\mathrm{p}=0.047$, but not between peak VO2 and transaortic velocity $(\mathrm{r}=0.117, \mathrm{p}=0.339)$ and peak $\mathrm{VO} 2$ and mean gradient $(\mathrm{r}=0.139, \mathrm{p}=0.256)$.

Over a median follow up time of 8 months (IQR 4 to 28 months), 28 patients (39\%) were referred for aortic valve intervention. There were 3 deaths. Logistic regression showed that aortic valve area and peak VO2 were not significant predictors for clinical events.

Conclusion Our data demonstrates that a significant proportion of patients with apparently asymptomatic aortic stenosis exhibit exercise limitation as measured by peak VO2. Cardiopulmonary exercise testing may provide incremental objective evidence of early deterioration, over and above parameters obtained from conventional treadmill testing, as suggested by current guidelines.

Conflict of Interest nil

\section{PREDICTIVE VALUE OF RIGHT VENTRICULAR IMPAIRMENT ON LONG-TERM SURVIVAL AFTER MITRAL VALVE SURGERY - COMPARISON OF MINI-MITRAL AND CONVENTIONAL SURGERY}

Amr Abdelrahman*, Maciej Debski, Syed Qadri, David Lai Chin kon, John Victor McGowan, Justin Tay, Joseph Zacharias, Kenneth Wong. Blackpool Victoria Hospital

\subsection{6/heartjnl-2019-BCS.130}

Introduction Right ventricular (RV) impairment may have prognostic value in patients undergoing mitral valve surgery. However, studies have yielded conflicting data. It is unclear whether RV dysfunction predicts long-term mortality, especially in mini-mitral era.

Methods We performed a retrospective analysis of consecutive patients referred from Blackpool, Blackburn, Chorley, Lancaster hospitals for mitral valve surgery between January 2013 and December 2015. The patients were followed-up for up to 6 years. Censor date was 22.02.2019. Survival data was obtained from hospital clinical system which is updated weekly with dates of death from UK's Office for National Statistics. Pre-operative transthoracic echocardiography (TTE) analysis was performed by an experienced echocardiographer who was blinded to the baseline characteristics, procedural data and outcome data. RV impairment was defined by reduced RV longitudinal function (TAPSE $<17 \mathrm{~mm}$ ) and dilated baseline RV diameter (RVD1 > $42 \mathrm{~mm}$ ). Primary outcome was all-cause mortality.

Results Of the 220 patients, $83(37.7 \%)$ were female. Mean age was $67.7 \pm 12.0$ years (range, 24-89). Minimally invasive (mini-mitral) approach was performed in 66 (30\%) and conventional (sternotomy) approach in 154 (70\%) patients. Concurrent CABG was done in $77(50 \%)$ patients in sternotomy group and in none of mini-mitral patients $(\mathrm{p}<0.001)$. Patients undergoing mini-mitral procedure compared to patients treated with conventional surgery were younger, less frequently had significant history of CAD, had lower EUROSCORE and more often were elective cases. Baseline characteristics are presented in table 1 . RV impairment was identified in preoperative TTE in $45(20.5 \%)$ patients. Patients with RV impairment were comparable to patients with preserved RV function in terms of all analysed variables (table 2). Mean follow-up duration was $4.0 \pm 1.6$, up to 6 years. $45(20.5 \%)$ patients died.

Mortality among patients with RV impairment was significantly higher than among patients with preserved RV function [log-rank $p=0.011)$. There was no significant difference in mortality between patients undergoing mini-mitral and conventional (log-rank $\mathrm{p}=0.346$ ). Patients with RV impairment had comparable survival irrespective of the type of surgical approach (log-rank $\mathrm{p}=0.707)$. RV impairment was associated with reduced survival following conventional surgery (figure 1), but did not statistically significantly influence.

Conclusion RV impairment was associated with reduced survival following conventional surgery, but did not statistically significantly influence survival after mini-mitral surgery. More studies are required to see if this is reproducible. If so, guidelines should include right ventricular impairment in decision making for surgical referral.

Conflict of Interest None

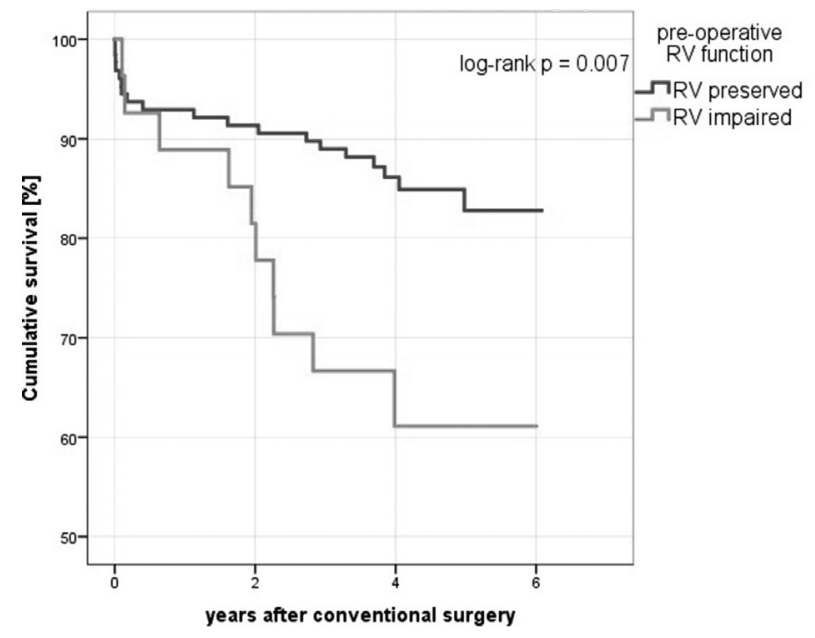

Abstract 133 Figure 1 Conventional mitral valve surgery

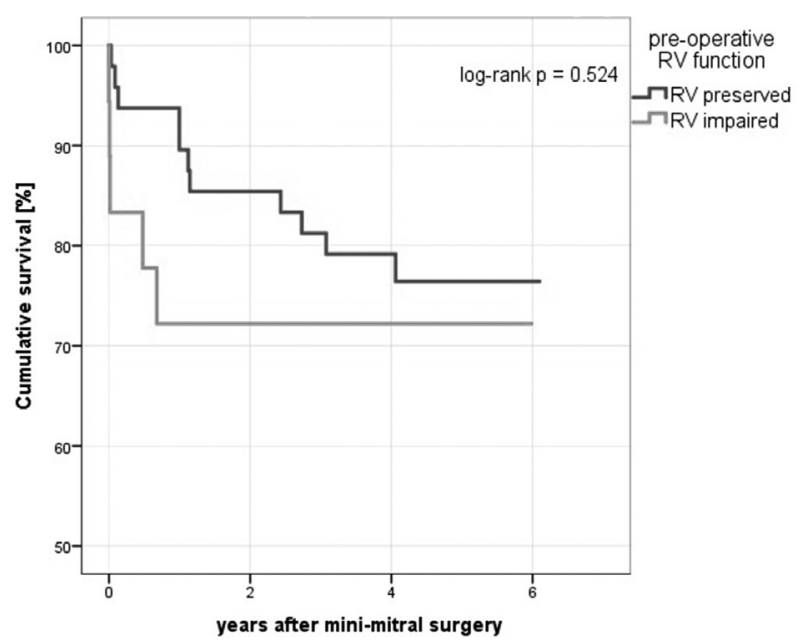

Abstract 133 Figure 2 Minimally invasive mitral valve surgery 